\title{
Exponential Stability and Periodicity of Fuzzy Delayed Reaction-Diffusion Cellular Neural Networks with Impulsive Effect
}

\author{
Guowei Yang, ${ }^{1}$ Yonggui Kao, ${ }^{2}$ and Changhong Wang ${ }^{2}$ \\ ${ }^{1}$ College of Information Engineering, Nanchang Hangkong University, Nanchang 330063, China \\ ${ }^{2}$ Space Control and Inertial Technology Research Center, Harbin Institute of Technology, Harbin, \\ Heilongjiang 150001, China
}

Correspondence should be addressed to Changhong Wang; chwang@hit.edu.cn

Received 3 September 2012; Accepted 4 January 2013

Academic Editor: Tingwen Huang

Copyright (C) 2013 Guowei Yang et al. This is an open access article distributed under the Creative Commons Attribution License, which permits unrestricted use, distribution, and reproduction in any medium, provided the original work is properly cited.

\begin{abstract}
This paper considers dynamical behaviors of a class of fuzzy impulsive reaction-diffusion delayed cellular neural networks (FIRDDCNNs) with time-varying periodic self-inhibitions, interconnection weights, and inputs. By using delay differential inequality, $M$-matrix theory, and analytic methods, some new sufficient conditions ensuring global exponential stability of the periodic FIRDDCNN model with Neumann boundary conditions are established, and the exponential convergence rate index is estimated. The differentiability of the time-varying delays is not needed. An example is presented to demonstrate the efficiency and effectiveness of the obtained results.
\end{abstract}

\section{Introduction}

The fuzzy cellular neural networks (FCNNs) model, which combines fuzzy logic with the structure of traditional neural networks (CNNs) [1-3], has been proposed by Yang et al. $[4,5]$. Unlike previous CNNs structures, the FCNNs model has fuzzy logic between its template and input and/or output besides the "sum of product" operation. Studies have shown that the FCNNs model is a very useful paradigm for image processing and pattern recognition [6-8]. These applications heavily depend on not only the dynamical analysis of equilibrium points but also on that of the periodic oscillatory solutions. In fact, the human brain is naturally in periodic oscillatory [9], and the dynamical analysis of periodic oscillatory solutions is very important in learning theory $[10,11]$, because learning usually requires repetition. Moreover, an equilibrium point can be viewed as a special periodic solution of neural networks with arbitrary period. Stability analysis problems for FCNNs with and without delays have recently been probed; see [12-22] and the references therein. Yuan et al. [13] have investigated stability of FCNNs by linear matrix inequality approach, and several criteria have been provided for checking the periodic solutions for FCNNs with timevarying delays. Huang [14] has probed exponential stability of fuzzy cellular neural networks with distributed delay, without considering reaction-diffusion effects.

Strictly speaking, reaction-diffusion effects cannot be neglected in both biological and man-made neural networks [19-32], especially when electrons are moving in noneven electromagnetic field. In [19], stability is considered for FCNNs with diffusion terms and time-varying delay. Wang and $\mathrm{Lu} \mathrm{[20]} \mathrm{have} \mathrm{probed} \mathrm{global} \mathrm{exponential} \mathrm{stability}$ of FCNNs with delays and reaction-diffusion terms. Song and Wang [21] have studied dynamical behaviors of fuzzy reaction-diffusion periodic cellular neural networks with variable coefficients and delays without considering pulsing effects. Wang et al. [22] have discussed exponential stability of impulsive stochastic fuzzy reaction-diffusion CohenGrossberg neural networks with mixed delays. Zhao and Mao [30] have investigated boundedness and stability of nonautonomous cellular neural networks with reaction-diffusion terms. Zhao and Wang [31] have considered existence of periodic oscillatory solution of reaction-diffusion neural networks with delays without fuzzy logic and impulsive effect. 
As we all know, many practical systems in physics, biology, engineering, and information science undergo abrupt changes at certain moments of time because of impulsive inputs [33]. Impulsive differential equations and impulsive neural networks have been received much interest in recent years; see, for example, [34-42] and the references therein. Yang and $\mathrm{Xu}$ [36] have investigated existence and exponential stability of periodic solution for impulsive delay differential equations and applications. $\mathrm{Li}$ and $\mathrm{Lu}$ [38] have discussed global exponential stability and existence of periodic solution of Hopfield-type neural networks with impulses without reaction-diffusion. To the best of our knowledge, few authors have probed the existence and exponential stability of the periodic solutions for the FIRDDCNN model with variable coefficients, and time-varying delays. As a result of the simultaneous presence of fuzziness, pulsing effects, reactiondiffusion phenomena, periodicity, variable coefficients and delays, the dynamical behaviors of this kind of model become much more complex and have not been properly addressed, which still remain important and challenging.

Motivated by the above discussion, we will establish some sufficient conditions for the existence and exponential stability of periodic solutions of this kind of FIRDDCNN model, applying delay differential inequality, $M$-matrix theory, and analytic methods. An example is employed to demonstrate the usefulness of the obtained results.

Notations. Throughout this paper, $\mathbb{R}^{n}$ and $\mathbb{R}^{n \times m}$ denote, respectively, the $n$-dimensional Euclidean space and the set of all $n \times m$ real matrices. The superscript " $\mathrm{T}$ " denotes matrix transposition and the notation $X \geq Y$ (resp., $X>Y$ ), where $X$ and $Y$ are symmetric matrices, means that $X-Y$ is positive semidefinite (resp., positive definite). $\Omega=\{x=$ $\left.\left(x_{1}, \ldots, x_{m}\right)^{\mathrm{T}},\left|x_{i}\right|<\mu\right\}$ is a bounded compact set in space $\mathbb{R}^{m}$ with smooth boundary $\partial \Omega$ and measure mes $\Omega>0$; Neumann boundary condition $\partial u_{i} / \partial n=0$ is the outer normal to $\partial \Omega ; L^{2}(\Omega)$ is the space of real functions $\Omega$ which are $L^{2}$ for the Lebesgue measure. It is a Banach space with the norm $\|u(t, x)\|_{2}=\left(\sum_{i=1}^{n}\left\|u_{i}(t, x)\right\|_{2}^{2}\right)^{1 / 2}$, where $u(t, x)=$ $\left(u_{1}(t, x), \ldots, u_{n}(t, x)\right)^{\mathrm{T}},\left\|u_{i}(t, x)\right\|_{2}=\left(\int_{\Omega}\left|u_{i}(t, x)\right|^{2} d x\right)^{1 / 2}$, $|u(t, x)|=\left(\left|u_{1}(t, x)\right|, \ldots,\left|u_{n}(t, x)\right|\right)^{\mathrm{T}}$. For function $g(x)$ with positive period $\omega$, we denote $\bar{g}=\max _{t \in[0, \omega]} g(t), \underline{g}=$ $\min _{t \in[0, \omega]} g(t)$. Sometimes, the arguments of a function or a matrix will be omitted in the analysis when no confusion can arise.

\section{Preliminaries}

Consider the impulsive fuzzy reaction-diffusion delayed cellular neural networks (FIRDDCNN) model:

$$
\begin{aligned}
\frac{\partial u_{i}(t, x)}{\partial t}= & \sum_{l=1}^{m} \frac{\partial}{\partial x_{l}}\left(D_{i} \frac{\partial u_{i}(t, x)}{\partial x_{l}}\right) \\
& -c_{i}(t) u_{i}(t, x)+\sum_{j=1}^{n} a_{i j}(t) f_{j}\left(u_{j}(t, x)\right)
\end{aligned}
$$

$$
\begin{aligned}
& +\sum_{j=1}^{n} b_{i j}(t) v_{j}(t)+J_{i}(t) \\
& +\bigwedge_{j=1}^{n} \alpha_{i j}(t) g_{j}\left(u_{j}\left(t-\tau_{j}(t), x\right)\right) \\
& +\bigvee_{j=1}^{n} \beta_{i j}(t) g_{j}\left(u_{j}\left(t-\tau_{j}(t), x\right)\right) \\
& +\bigwedge_{j=1}^{n} T_{i j}(t) v_{j}(t)+\bigvee_{j=1}^{n} H_{i j}(t) v_{j}(t), \\
& u_{i}\left(t_{k}^{+}, x\right)-u_{i}\left(t_{k}^{-}, x\right)=I_{i k}\left(u_{i}\left(t_{k}^{-}, x\right)\right), \\
& t=t_{k}, \quad k \in \mathbb{Z}_{+}, x \in \Omega, \\
& \quad \frac{\partial u_{i}(t, x)}{\partial n}=0, \quad t \geq t_{0}, x \in \partial \Omega, \\
& u_{i}\left(t_{0}+s, x\right)=\psi_{i}(s, x), \quad-\tau_{j} \leq s \leq 0, x \in \Omega,
\end{aligned}
$$

where $n \geq 2$ is the number of neurons in the network and $u_{i}(t, x)$ corresponds to the state of the $i$ th neuron at time $t$ and in space $x ; D=\operatorname{diag}\left(D_{1}, D_{2}, \ldots, D_{n}\right)$ is the diffusionmatrix and $D_{i} \geq 0 ; \Delta=\sum_{k=1}^{m}\left(\partial^{2} / \partial x_{k}^{2}\right)$ is the Laplace operator; $f_{j}\left(u_{j}(t, x)\right)$ denotes the activation function of the $j$ th unit and $v_{j}(t)$ the activation function of the $j$ th unit; $J_{i}(t)$ is an input at time $t ; c_{i}(t)>0$ represents the rate with which the $i$ th unit will reset its potential to the resting state in isolation when disconnected from the networks and external inputs at time $t ; a_{i j}(t)$ and $b_{i j}(t)$ are elements of feedback template and feed forward template at time $t$, respectively. Moreover, in model (1), $\alpha_{i j}(t), \beta_{i j}(t), T_{i j}(t)$, and $H_{i j}(t)$ are elements of fuzzy feedback MIN template, fuzzy feedback MAX template, fuzzy feed forward MIN template, and fuzzy feed forward MAX template at time $t$, respectively; the symbols " $\wedge$ " and " $\bigvee$ " denote the fuzzy AND and fuzzy OR operation, respectively; time-varying delay $\tau_{j}(t)$ is the transmission delay along the axon of the $j$ th unit and satisfies $0 \leq \tau_{j}(t) \leq \tau_{j}\left(\tau_{j}\right.$ is a constant); the initial condition $\phi_{i}(s, x)$ is bounded and continuous on $[-\tau, 0] \times \Omega$, where $\tau=\max _{1 \leq j \leq n} \tau_{j}$. The fixed moments $t_{k}$ satisfy $0=t_{0}<t_{1}<t_{2} \ldots, \lim _{k \rightarrow+\infty} t_{k}=$ $+\infty, k \in \mathbb{N} . u_{i}\left(t_{k}^{+}, x\right)$ and $u_{i}\left(t_{k}^{-}, x\right)$ denote the right-hand and left-hand limits at $t_{k}$, respectively. We always assume $u_{i}\left(t_{k}^{+}, x\right)=u_{i}\left(t_{k}, x\right)$, for all $k \in N$. The initial value functions $\psi(s, x)$ belong to $\mathrm{PC}_{\Omega}\left([-\tau, 0] \times \Omega ; R^{n}\right)$. $\mathrm{PC}_{\Omega}\left(J \times \Omega, L^{2}(\Omega)\right)=$ $\left\{\psi: J \times \Omega \rightarrow L^{2}(\Omega) \mid\right.$ for every $t \in J, \psi(t, x) \in L^{2}(\Omega)$; for any fixed $x \in \Omega, \psi(t, x)$ is continuous for all but at most countable points $s \in J$ and at these points, $\psi\left(s^{+}, x\right)$ and $\psi\left(s^{-}, x\right)$ exist, $\left.\psi\left(s^{+}, x\right)=\psi\left(s^{-}, x\right)\right\}$, where $\psi\left(s^{+}, x\right)$ and $\psi\left(s^{-}, x\right)$ denote the right-hand and left-hand limit of the function $\psi(s, x)$, respectively. Especially, let $\mathrm{PC}_{\Omega}=\operatorname{PC}\left([-\tau, 0] \times \Omega, L^{2}(\Omega)\right)$. For any $\psi(t, x)=\left(\psi_{1}(t, x), \ldots, \psi_{n}(t, x)\right) \in \mathrm{PC}_{\Omega}$, suppose that $\left|\psi_{i}(t, x)\right|_{\tau}=\sup _{-\tau<s \leq 0}\left|\psi_{i}(t+s, x)\right|$ exists as a finite number 
and introduce the norm $\|\psi(t)\|_{2}=\left(\sum_{i=1}^{n}\left\|\psi_{i}(t)\right\|_{2}^{2}\right)^{1 / 2}$, where $\left\|\psi_{i}(t)\right\|_{2}=\left(\int_{\Omega}\left|\psi_{i}(t, x)\right|^{2} d x\right)^{1 / 2}$.

Throughout the paper, we make the following assumptions.

(H1) There exists a positive diagonal matrix $F=$ $\operatorname{diag}\left(F_{1}, F_{2}, \ldots, F_{n}\right)$, and $G=\operatorname{diag}\left(G_{1}, G_{2}, \ldots, G_{n}\right)$ such that

$$
\begin{aligned}
& F_{j}=\sup _{x \neq y}\left|\frac{f_{j}(x)-f_{j}(y)}{x-y}\right|, \\
& G_{j}=\sup _{x \neq y}\left|\frac{g_{j}(x)-g_{j}(y)}{x-y}\right|
\end{aligned}
$$

for all $x \neq y, j=1,2, \ldots, n$.

$(\mathbf{H} 2) c_{i}(t)>0, a_{i j}(t), b_{i j}(t), \alpha_{i j}(t), \beta_{i j}(t), T_{i j}(t), H_{i j}(t), v_{i}(t)$, $I_{i}(t)$, and $\tau_{j}(t) \geq 0$ are periodic function with a common positive period $\omega$ for all $t \geq t_{0}, i, j=1$, $2, \ldots, n$.

(H3) For $\omega>0, i=1,2, \ldots, n$, there exists $q \in Z_{+}$such that $t_{k}+\omega=t_{k+q}, I_{i k}\left(u_{i}\right)=I_{i(k+q)}\left(u_{i}\right)$ and $I_{i k}\left(u_{i}\left(t_{k}, x\right)\right)$ are Lipschitz continuous in $\mathbb{R}^{n}$.

Definition 1. The model in (1) is said to be globally exponentially periodic if (i) there exists one $\omega$-periodic solution and (ii) all other solutions of the model converge exponentially to it as $t \rightarrow+\infty$.

Definition 2 (see [26]). Let $\mathbf{C}=\left([t-\tau, t], \mathbb{R}^{n}\right)$, where $\tau \geq$ 0 and $F(t, x, y) \in \mathbf{C}\left(\mathbb{R}^{+} \times \mathbb{R}^{n} \times \mathbf{C}, \mathbb{R}^{n}\right)$. Then the function $F(t, x, y)=\left(f_{1}(t, x, y), f_{2}(t, x, y), \ldots, f_{n}(t, x, y)\right)^{\mathrm{T}}$ is called an $M$-function, if (i) for every $t \in \mathbb{R}^{+}, x \in \mathbb{R}^{n}, y^{(1)} \in \mathbf{C}$, there holds $F\left(t, x, y^{(1)}\right) \leq F\left(t, x, y^{(2)}\right)$, for $y^{(1)} \leq y^{(2)}$, where $y^{(1)}=\left(y_{1}^{(1)}, \ldots, y_{n}^{(1)}\right)^{\mathrm{T}}$ and $y^{(2)}=\left(y_{1}^{(2)}, \ldots, y_{n}^{(2)}\right)^{\mathrm{T}}$; (ii) every $i$ th element of $F$ satisfies $f_{i}\left(t, x^{(1)}, y\right) \leq f_{i}\left(t, x^{(2)}, y\right)$ for any $y \in \mathbf{C}, t \geq t_{0}$, where arbitrary $x^{(1)}$ and $x^{(2)}\left(x^{(1)} \leq x^{(2)}\right)$ belong to $\mathbb{R}^{n}$ and have the same $i$ th component $x_{i}^{(1)}=x_{i}^{(2)}$. Here, $x^{(1)}=\left(x_{1}^{(1)}, \ldots, x_{n}^{(1)}\right)^{\mathrm{T}}, x^{(2)}=\left(x_{1}^{(2)}, \ldots, x_{n}^{(2)}\right)^{\mathrm{T}}$.

Definition 3 (see [26]). A real matrix $A=\left(a_{i j}\right)_{n \times n}$ is said to be a nonsingular $M$-matrix if $a_{i j} \leq 0 \quad(i \neq j ; i, j=1, \ldots, n)$ and all successive principal minors of $A$ are positive.

Lemma 4 (see [13]). Let $u$ and $u^{*}$ be two states of the model in (1), then we have

$$
\begin{array}{r}
\left|\bigwedge_{j=1}^{n} \alpha_{i j}(t) f_{j}\left(u_{j}\right)-\bigwedge_{j=1}^{n} \alpha_{i j}(t) f_{j}\left(u_{j}^{*}\right)\right| \\
\leq \sum_{j=1}^{n}\left|\alpha_{i j}(t)\right| \cdot\left|f_{j}\left(u_{j}\right)-f_{j}\left(u_{j}^{*}\right)\right|,
\end{array}
$$

$$
\begin{array}{r}
\left|\bigvee_{j=1}^{n} \beta_{i j}(t) f_{j}\left(u_{j}\right)-\bigvee_{j=1}^{n} \beta_{i j}(t) f_{j}\left(u_{j}^{*}\right)\right| \\
\leq \sum_{j=1}^{n}\left|\beta_{i j}(t)\right| \cdot\left|f_{j}\left(u_{j}\right)-f_{j}\left(u_{j}^{*}\right)\right| .
\end{array}
$$

Lemma 5 (see [26]). Assume that $F(t, x, y)$ is an $M$-function, and (i) $x(t)<y(t), t \in\left[t-\tau, t_{0}\right]$, (ii) $D^{+} y(t)>$ $F\left(t, y(t), y^{s}(t)\right), D^{+} x(t) \leq F\left(t, x(t), x^{s}(t)\right), t \geq t_{0}$, where $x^{s}(t)=\sup _{-\tau \leq s \leq 0} x(t+s), y^{s}(t)=\sup _{-\tau \leq s \leq 0} y(t+s)$. Then $x(t)<y(t), t \geq t_{0}$.

\section{Main Results and Proofs}

We should first point out that, under assumptions (H1), (H2), and (H3), the FIRDDCNN model (1) has at least one $\omega$-periodic solution of [26]. The proof of the existence of the $\omega$-periodic solution of (1) can be carried out similar to $[26,28]$ by the nonlinear functional analysis methods such as topological degree and here is omitted. We will mainly discuss the uniqueness of the periodic solution and its exponential stability.

Theorem 6. Assume that (H1)-(H3) holds. Furthermore, assume that the following conditions hold

(H4) $\underline{C}-\bar{A} F-(\bar{\alpha}+\bar{\beta}) G$ is a nonsingular M-matrix.

(H5) The impulsive operators $h_{k}(u)=u+I_{k}(u)$ is Lipschitz continuous in $\mathbb{R}^{n}$; that is, there exists a nonnegative diagnose matrix $\Gamma_{k}=\operatorname{diag}\left(\gamma_{1 \mathrm{k}}, \ldots, \gamma_{\mathrm{nk}}\right)$ such that $\left|h_{k}(u)-h_{k}\left(u^{*}\right)\right| \leq \Gamma_{k}\left|u-u^{*}\right|$ for all $u, u^{*} \in \mathbb{R}^{n}$, $k \in N^{+}$, where $\left|h_{k}(u)\right|=\left(\left|h_{1 k}\left(u_{1}\right)\right|, \ldots,\left|h_{n k}\left(u_{n}\right)\right|\right)^{\mathrm{T}}$, $I_{k}(u)=\left(I_{1 k}\left(u_{1}\right), \ldots, I_{n k}\left(u_{n}\right)\right)^{\mathrm{T}}$.

(H6) $\eta=\sup _{k \in N^{+}}\left\{\ln \eta_{k} /\left(t_{k}-t_{k-1}\right)\right\}<\lambda$, where $\eta_{k}=$ $\max _{1 \leq i \leq n}\left\{1, \gamma_{i k}\right\}, k \in N^{+}$.

Then the model (1) is global exponential periodic and the exponential convergence rate index $\lambda-\eta$ and $\lambda$ can be estimated by

$$
\begin{array}{r}
\xi_{i}\left(\lambda-\underline{c}_{i}\right) \\
+\sum_{j=1}^{n} \xi_{j}\left(\left|\bar{a}_{i j}\right| F_{j}+e^{\tau \lambda}\left(\left|\bar{\alpha}_{i j}\right|+\left|\bar{\beta}_{i j}\right|\right) G_{j}\right)<0 \\
i=1, \ldots, n,
\end{array}
$$

where $\underline{C}=\operatorname{diag}\left(\underline{c}_{1}, \ldots, \underline{c}_{n}\right)$ and $\xi_{i}>0, \bar{A}=\left(\left|\bar{a}_{i j}\right|\right)_{n \times n}, \quad \bar{\alpha}=$ $\left(\left|\bar{\alpha}_{i j}\right|\right)_{n \times n}, \bar{\beta}=\left(\left|\bar{\beta}_{i j}\right|\right)_{n \times n}$, satisfies $-\xi_{i} \underline{c}_{i}+\sum_{j=1}^{n} \xi_{i}\left(\left|\bar{a}_{i j}\right| F_{i}+\right.$ $\left.\left(\left|\bar{\alpha}_{i j}\right|+\left|\bar{\beta}_{i j}\right|\right) G_{i}\right)<0$.

Proof. For any $\phi, \psi \in \mathrm{PC}_{\Omega}$, let $u(t, x, \phi)=\left(u_{1}(t, x, \phi), \ldots\right.$, $\left.u_{n}(t, x, \phi)\right)^{\mathrm{T}}$ be a periodic solution of the system (1) starting 
from $\phi$ and $u(t, x, \psi)=\left(u_{1}(t, x, \psi), \ldots, u_{n}(t, x, \psi)\right)^{\mathrm{T}}$, a solution of the system (1) starting from $\psi$. Define

$$
\begin{gathered}
u_{t}(\phi, x)=u(t+s, x, \phi), \\
u_{t}(\psi, x)=u(t+s, x, \psi), \quad s \in[-\tau, 0],
\end{gathered}
$$

and we can see that $u_{t}(\phi, x), u_{t}(\psi, x) \in \mathrm{PC}_{\Omega}$ for all $t>0$. Let $U_{i}=u_{i}(t, x, \phi)-u_{i}(t, x, \psi)$, then from (1) we get

$$
\begin{aligned}
\frac{\partial U_{i}}{\partial t}=\sum_{l=1}^{m} \frac{\partial}{\partial x_{l}}\left(D_{i} \frac{\partial U_{i}}{\partial x_{l}}\right)-c_{i}(t) U_{i}+\sum_{j=1}^{n} a_{i j}(t) \\
\times\left[f_{j}\left(u_{j}(t, x, \phi)\right)-f_{j}\left(u_{j}(t, x, \psi)\right)\right] \\
+\left[\bigwedge_{j=1}^{n} \alpha_{i j}(t) f_{j}\left(u_{j}\left(t-\tau_{j}(t), x, \phi\right)\right)\right. \\
\left.\quad-\bigwedge_{j=1}^{n} \alpha_{i j}(t) f_{j}\left(u_{j}\left(t-\tau_{j}(t), x, \psi\right)\right)\right] \\
+\left[\bigvee_{j=1}^{n} \beta_{i j}(t) f_{j}\left(u_{j}\left(t-\tau_{j}(t), x, \phi\right)\right)\right. \\
\left.\quad-\bigvee_{j=1}^{n} \beta_{i j}(t) f_{j}\left(u_{j}\left(t-\tau_{j}(t), x, \psi\right)\right)\right]
\end{aligned}
$$

for all $t \neq t_{k}, x \in \Omega, i=1, \ldots, n$.

Multiplying both sides of (6) by $U_{i}$ and integrating it in $\Omega$, we have

$$
\begin{aligned}
& \frac{1}{2} \frac{\mathrm{d}}{\mathrm{d} t} \int_{\Omega} U_{i}^{2} \mathrm{~d} x \\
& =\int_{\Omega} U_{i} \sum_{l=1}^{m} \frac{\partial}{\partial x_{l}}\left(D_{i} \frac{\partial U_{i}}{\partial x_{l}}\right) \mathrm{d} x \\
& \quad-c_{i}(t) \int_{\Omega} U_{i}^{2} d x+\sum_{j=1}^{n} a_{i j}(t) \int_{\Omega} U_{i} \\
& \quad \times\left[f_{j}\left(u_{j}(t, x, \phi)-f_{j}\left(u_{j}(t, x, \psi)\right)\right)\right] \mathrm{d} x \\
& \quad+\int_{\Omega} U_{i}\left[\bigwedge_{j=1}^{n} \alpha_{i j}(t) f_{j}\left(u_{j}\left(t-\tau_{j}(t), x, \phi\right)\right)\right. \\
& \quad+\int_{\Omega} U_{i}\left[\bigvee_{j=1}^{n} \beta_{i j}(t) f_{j}\left(u_{j}\left(t-\tau_{j}(t), x, \phi\right)\right)\right. \\
& \left.\quad-\bigvee_{i j}^{n} \beta_{i j}(t) f_{j}\left(u_{j}\left(t-\tau_{j}(t), x, \psi\right)\right)\right] \mathrm{d} x
\end{aligned}
$$

for $t \neq t_{k}, x \in \Omega, i=1, \ldots, n$. By boundary condition and Green Formula, we can get

$$
\int_{\Omega} U_{i} \sum_{l=1}^{m} \frac{\partial}{\partial x_{l}}\left(D_{i} \frac{\partial U_{i}}{\partial x_{l}}\right) d x \leq-D_{i} \int_{\Omega}\left(\nabla U_{i}\right)^{2} \mathrm{~d} x .
$$

Then, from (8), (9), (H1)-(H2), Lemma 4, and the Holder inequality,

$$
\begin{aligned}
& \frac{d}{d t}\left\|U_{i}\right\|_{2}^{2} \\
& \leq-2 c_{i}\left\|U_{i}\right\|_{2}^{2}+2 \sum_{j=1}^{n}\left|\bar{a}_{i j}\right| F_{j}\left\|U_{i}\right\|_{2}\left\|U_{j}\right\|_{2} \\
& \quad+2 \sum_{j=1}^{n}\left(\left|\bar{\alpha}_{i j}\right|+\left|\bar{\beta}_{i j}\right|\right) G_{j}\left\|U_{i}\right\|_{2} \\
& \quad \times\left\|u_{j}\left(t-\tau_{j}(t), x, \phi\right)-u_{j}\left(t-\tau_{j}(t), x, \psi\right)\right\|_{2}, \\
& t \neq t_{k} .
\end{aligned}
$$

Thus,

$$
\begin{aligned}
& D^{+}\left\|U_{i}\right\|_{2} \\
& \leq-\underline{c}_{i}\left\|U_{i}\right\|_{2}+\sum_{j=1}^{n}\left|\bar{\alpha}_{i j}\right| F_{j}\left\|U_{j}\right\|_{2} \\
& \quad+\sum_{j=1}^{n}\left(\left|\bar{\alpha}_{i j}\right|+\left|\bar{\beta}_{i j}\right|\right) G_{j} \\
& \quad \times\left\|u_{j}\left(t-\tau_{j}(t), x, \phi\right)-u_{j}\left(t-\tau_{j}(t), x, \psi\right)\right\|_{2}, \\
& t \neq t_{k}
\end{aligned}
$$

for $i=1, \ldots, n$. Since $\underline{C}-(\bar{A} F+(\bar{\alpha}+\bar{\beta}) G)$ is a nonsingular $M$-matrix, there exists a vector $\xi=\left(\xi_{1}, \ldots, \xi_{n}\right)^{\mathrm{T}}>0$ such that

$$
-\xi_{i} \underline{c}_{i}+\sum_{j=1}^{n} \xi_{j}\left(\left|\bar{a}_{i j}\right| F_{j}+\left(\left|\bar{\alpha}_{i j}\right|+\left|\bar{\beta}_{i j}\right|\right) G_{j}\right)<0 .
$$

Considering functions

$$
\begin{array}{r}
\Psi_{i}(y)=\xi_{i}\left(y-\underline{c}_{i}\right) \\
+\sum_{j=1}^{n} \xi_{j}\left(\left|\bar{a}_{i j}\right| F_{j}+e^{\tau y}\left(\left|\bar{\alpha}_{i j}\right|+\left|\bar{\beta}_{i j}\right|\right) G_{j}\right), \\
i=1, \ldots, n,
\end{array}
$$

we know from (11) that $\Psi_{i}(0)<0$ and $\Psi_{i}(y)$ is continuous. Since $\mathrm{d} \Psi_{i}(y) / \mathrm{d} y>0, \Psi_{i}(y)$ is strictly monotonically increasing, there exists a scalar $\lambda_{i}>0$ such that

$$
\begin{aligned}
\Psi_{i}\left(\lambda_{i}\right)= & \xi_{i}\left(\lambda_{i}-\underline{c}_{i}\right) \\
& +\sum_{j=1}^{n} \xi_{j}\left(\left|\bar{a}_{i j}\right| F_{j}+e^{\tau \lambda_{i}}\left(\left|\bar{\alpha}_{i j}\right|+\left|\bar{\beta}_{i j}\right|\right) G_{j}\right)=0,
\end{aligned}
$$


Choosing $0<\lambda<\min \left\{\lambda_{1}, \ldots, \lambda_{n}\right\}$, we have

$$
\begin{array}{r}
\xi_{i}\left(\lambda_{i}-\underline{c}_{i}\right) \\
+\sum_{j=1}^{n} \xi_{j}\left(\left|\bar{a}_{i j}\right| F_{j}+e^{\tau \lambda_{i}}\left(\left|\bar{\alpha}_{i j}\right|+\left|\bar{\beta}_{i j}\right|\right) G_{j}\right)<0 \\
i=1, \ldots, n .
\end{array}
$$

That is,

$$
\lambda \xi-(\underline{C}-\bar{A} F) \xi+(\bar{\alpha}+\bar{\beta}) G \xi e^{-\lambda t}<0 .
$$

Furthermore, choose a positive scalar $p$ large enough such that

$$
p e^{-\lambda t} \xi>(1,1, \ldots, 1)^{\mathrm{T}}, \quad t \in[-\tau, 0] .
$$

For any $\varepsilon>0$, let

$$
r(t)=p e^{-\lambda t}\left(\|\phi-\psi\|_{2}+\varepsilon\right) \xi, \quad t_{0} \leq t<t_{1} .
$$

From (15)-(17), we obtain

$$
\begin{aligned}
D^{+} r(t) & >-(\underline{C}-\bar{A} F) r(t)+(\bar{\alpha}+\bar{\beta}) G r^{s}(t) \\
& =: V\left(t, r(t), r^{s}(t)\right), \quad t_{0} \leq t<t_{1},
\end{aligned}
$$

where $r^{s}(t)=\left(r_{1}^{s}(t), \ldots, r_{n}^{s}(t)\right)^{\mathrm{T}}$ and $r_{i}^{s}(t)=\sup _{-\tau \leq s \leq 0} p e^{-\lambda(t+s)}$ $\left(\|\phi-\varphi\|_{2}+\varepsilon\right) \xi_{i}$. It is easy to verify that $V\left(t, r(t), r^{s}(t)\right)$ is an $M$-function. It follows also from (16) and (17) that

$$
\begin{aligned}
\left\|U_{i}\right\|_{2} & \leq\|\phi-\varphi\|_{2}<p e^{-\lambda t} \xi_{i}\|\phi-\varphi\|_{2} \\
& <r_{i}(t), \quad t \in[-\tau, 0], i=1,2, \ldots, n .
\end{aligned}
$$

Denote

$$
\begin{aligned}
U^{\diamond}:= & \left(\left\|u_{1}(t, x, \phi)-u_{1}(t, x, \psi)\right\|_{2}, \ldots,\right. \\
& \left.\left\|u_{n}(t, x, \phi)-u_{n}(t, x, \psi)\right\|_{2}\right)^{\mathrm{T}}, \\
U^{\diamond(s)}:= & \left(\left\|u_{1}(t, x, \phi)-u_{1}(t, x, \psi)\right\|_{2}^{(s)}, \ldots,\right. \\
& \left.\left\|u_{n}(t, x, \phi)-u_{n}(t, x, \psi)\right\|_{2}^{(s)}\right)^{\mathrm{T}},
\end{aligned}
$$

where $\left\|U_{i}\right\|_{2}^{(s)}=\sup _{-\tau \leq s \leq 0}\left\|u_{i}(t+s, x, \phi)-u_{i}(t+s, x, \psi)\right\|_{2}$, then

$$
U^{\diamond}<r(t), \quad t \in[-\tau, 0] .
$$

From (10), we can obtain

$$
\begin{aligned}
D^{+} U^{\diamond} & \leq-(\underline{C}-\bar{A} F) U^{\diamond}+(\bar{\alpha}+\bar{\beta}) G U^{\diamond(s)} \\
& =V\left(t, U^{\diamond}, U^{\diamond(s)}\right), \quad t \neq t_{k} .
\end{aligned}
$$

Now, it follows from (18)-(22) and Lemma 5 that

$$
\begin{array}{r}
U^{\diamond}<r(t)=p e^{-\lambda t}\left(\|\phi-\psi\|_{2}+\varepsilon\right) \xi, \\
t_{0} \leq t<t_{1} .
\end{array}
$$

Letting $\varepsilon \rightarrow 0$, we have

$$
U^{\diamond} \leq p \xi\|\phi-\psi\|_{2} e^{-\lambda t}, \quad t_{0} \leq t<t_{1} .
$$

And moreover, from (24), we get

$$
\left(\sum_{i=1}^{n}\left\|U_{i}\right\|_{2}^{2}\right)^{1 / 2} \leq p\left(\sum_{i=1}^{n} \xi_{i}^{2}\right)^{1 / 2}\|\phi-\psi\|_{2} e^{-\lambda t},
$$

$$
t_{0} \leq t<t_{1} \text {. }
$$

Let $\widetilde{M}=p\left(\sum_{i=1}^{n} \xi_{i}^{2}\right)^{1 / 2}$, then $\widetilde{M} \geq 1$. Define $W(t)=\| u_{t}(x, \phi)-$ $u_{t}(x, \psi) \|_{2}$; it follows from (25) and the definitions of $u_{t}(\phi, x)$ and $u_{t}(\psi, x)$ that

$$
\begin{aligned}
W(t) & =\left\|u_{t}(x, \phi)-u_{t}(x, \psi)\right\|_{2} \\
& \leq \widetilde{M}\|\phi-\psi\|_{2} e^{-\lambda t}, \quad t_{0} \leq t<t_{1} .
\end{aligned}
$$

It is easily observed that

$$
W(t) \leq \widetilde{M}\|\phi-\psi\|_{2} e^{-\lambda t}, \quad-\tau \leq t \leq t_{0}=0 .
$$

Because (26) holds, we can suppose that for $l \leq k$ inequality

$$
\begin{array}{r}
W(t) \leq \eta_{0} \cdots \eta_{l-1} \widetilde{M}\|\phi-\psi\|_{2} e^{-\lambda t}, \\
t_{l-1} \leq t<t_{l}
\end{array}
$$

holds, where $\eta_{0}=1$. When $l=k+1$, we note (H5) that

$$
\begin{aligned}
W\left(t_{k}\right) & =\left\|u_{t_{k}}(x, \phi)-u_{t_{k}}(x, \psi)\right\|_{2} \\
& =\left\|h_{k}\left(u_{t_{k}}^{-}(x, \phi)\right)-h_{k}\left(u_{t_{k}}^{-}(x, \psi)\right)\right\|_{2} \\
& \leq \rho\left(\Gamma_{k}^{2}\right)\left\|u_{t_{k}}^{-}(x, \phi)-u_{t_{k}}^{-}(x, \psi)\right\|_{2} \\
& =\rho\left(\Gamma_{k}^{2}\right) W\left(t_{k}^{-}\right) \\
& \leq \eta_{0} \cdots \eta_{l-1} \rho\left(\Gamma_{k}^{2}\right) \widetilde{M}\|\phi-\psi\|_{2} e^{-\lambda t_{k}} \\
& \leq \eta_{0} \cdots \eta_{l-1} \eta_{k} \rho\left(\Gamma_{k}^{2}\right) \widetilde{M}\|\phi-\psi\|_{2} e^{-\lambda t_{k}},
\end{aligned}
$$

where $\rho\left(\Gamma_{k}^{2}\right)$ is the spectral radius of $\Gamma_{k}^{2}$. Let $M=$ $\max \left\{\widetilde{M}, \rho\left(\Gamma_{k}^{2}\right) \widetilde{M}\right\}$, by (28), (29), and $\eta \geq 1$, we obtain

$$
\begin{array}{r}
W(t) \leq \eta_{0} \cdots \eta_{l-1} \eta_{k} M\|\phi-\psi\|_{2} e^{-\lambda t}, \\
t_{k}-\tau \leq t \leq t_{k} .
\end{array}
$$

Combining (10), (17), (30), and Lemma 5, we get

$$
\begin{array}{r}
W(t) \leq \eta_{0} \cdots \eta_{l-1} \eta_{k} M\|\phi-\psi\|_{2} e^{-\lambda t}, \\
t_{k} \leq t<t_{k+1}, \quad k \in N^{+} .
\end{array}
$$

Applying mathematical induction, we conclude that

$$
\begin{array}{r}
W(t) \leq \eta_{0} \cdots \eta_{l-1} M\|\phi-\psi\|_{2} e^{-\lambda t}, \\
t_{k-1} \leq t<t_{k}, k \in N^{+} .
\end{array}
$$


From (H6) and (32), we have

$$
\begin{aligned}
& W(t) \leq e^{\eta t_{1}} e^{\eta\left(t_{2}-t_{1}\right)} \cdots e^{\eta\left(t_{k-1}-t_{k-2}\right)} \\
& \times M\|\phi-\psi\|_{2} e^{-\lambda t} \leq M\|\phi-\psi\|_{2} e^{\eta t} e^{-\lambda t} \\
&= M\|\phi-\psi\|_{2} e^{-(\lambda-\eta) t}, \\
& t_{k-1} \leq t<t_{k}, k \in N^{+} .
\end{aligned}
$$

This means that

$$
\begin{aligned}
& \left\|u_{t}(x, \phi)-u_{t}(x, \psi)\right\|_{2} \\
& \quad \leq M\|\phi-\psi\|_{2} e^{-(\lambda-\eta) t} \\
& \quad \leq M\|\phi-\psi\|_{2} e^{-(\lambda-\eta)(t-\tau)}, \quad t \geq t_{0},
\end{aligned}
$$

choosing a positive integer $N$ such that

$$
M e^{-(\lambda-\eta)(N \omega-\tau)} \leq \frac{1}{6} .
$$

Define a Poincare mapping $\mathfrak{D}: \Gamma \rightarrow \Gamma$ by

$$
\mathfrak{D}(\phi)=u_{\omega}(x, \phi),
$$

Then

$$
\mathfrak{D}^{N}(\phi)=u_{N \omega}(x, \phi) .
$$

Setting $t=N \omega$ in (34), from (35) and (37), we have

$$
\left\|\mathfrak{D}^{N}(\phi)-\mathfrak{D}^{N}(\psi)\right\|_{2} \leq \frac{1}{6}\|\phi-\psi\|_{2}
$$

which implies that $\mathfrak{D}^{N}$ is a contraction mapping. Thus, there exists a unique fixed point $\phi^{*} \in \Gamma$ such that

$$
\mathfrak{D}^{N}\left(\mathfrak{D}\left(\phi^{*}\right)\right)=\mathfrak{D}\left(\mathfrak{D}^{N}\left(\phi^{*}\right)\right)=\mathfrak{D}\left(\phi^{*}\right) .
$$

From (37), we know that $\mathfrak{D}\left(\phi^{*}\right)$ is also a fixed point of $\mathfrak{D}^{N}$, and then it follows from the uniqueness of the fixed point that

$$
\mathfrak{D}\left(\phi^{*}\right)=\phi^{*}, \quad \text { that is, } u_{\omega}\left(x, \phi^{*}\right)=\phi^{*} \text {. }
$$

Let $u\left(t, x, \phi^{*}\right)$ be a solution of the model (1), then $u(t+$ $\left.\omega, x, \phi^{*}\right)$ is also a solution of the model (1). Obviously,

$$
u_{t+\omega}\left(x, \phi^{*}\right)=u_{t}\left(u_{\omega}\left(x, \phi^{*}\right)\right)=u_{t}\left(x, \phi^{*}\right),
$$

for all $t \geq t_{0}$. Hence, $u\left(t+\omega, x, \phi^{*}\right)=u\left(t, x, \phi^{*}\right)$, which shows that $u\left(t, x, \phi^{*}\right)$ is exactly one $\omega$-periodic solution of model (1). It is easy to see that all other solutions of model (1) converge to this periodic solution exponentially as $t \rightarrow+\infty$, and the exponential convergence rate index is $\lambda-\eta$. The proof is completed.

Remark 7. When $c_{i}(t)=c_{i}, a_{i j}(t)=a_{i j}, b_{i j}(t)=b_{i j}, \alpha_{i j}(t)=\alpha_{i j}$, $\beta_{i j}(t)=\beta_{i j}, T_{i j}(t)=T_{i j}, H_{i j}(t)=H_{i j}, v_{i}(t)=v_{i}, I_{i}(t)=I_{i}$, and $\tau_{t}=\tau_{i}\left(c_{i}, a_{i j}, b_{i j}, \alpha_{i j}, \beta_{i j}, T_{i j}, H_{i j}, v_{i}, I_{i}\right.$, and $\tau_{i}$ are constants $)$, then the model (1) is changed into

$$
\begin{aligned}
& \frac{\partial u_{i}(t, x)}{\partial t}=\sum_{l=1}^{m} \frac{\partial}{\partial x_{l}}\left(D_{i} \frac{\partial u_{i}(t, x)}{\partial x_{l}}\right) \\
& -c_{i} u_{i}(t, x)+\sum_{j=1}^{n} a_{i j} f_{j}\left(u_{j}(t, x)\right) \\
& +\sum_{j=1}^{n} b_{i j} v_{j}+J_{i} \\
& +\bigwedge_{j=1}^{n} \alpha_{i j} g_{j}\left(u_{j}\left(t-\tau_{j}(t), x\right)\right) \\
& +\bigvee_{j=1}^{n} \beta_{i j} g_{j}\left(u_{j}\left(t-\tau_{j}(t), x\right)\right) \\
& +\bigwedge_{j=1}^{n} T_{i j} v_{j}+\bigvee_{j=1}^{n} H_{i j} v_{j}, \quad t \neq t_{k}, x \in \Omega, \\
& u_{i}\left(t_{k}^{+}, x\right)-u_{i}\left(t_{k}^{-}, x\right)=I_{i k}\left(u_{i}\left(t_{k}^{-}, x\right)\right) \text {, } \\
& t=t_{k}, k \in \mathbb{Z}_{+}, x \in \Omega, \\
& \frac{\partial u_{i}(t, x)}{\partial n}=0, \quad t \geq t_{0}, x \in \partial \Omega \\
& u_{i}\left(t_{0}+s, x\right)=\psi_{i}(s, x), \quad-\tau_{j} \leq s \leq 0, x \in \Omega \text {. }
\end{aligned}
$$

For any positive constant $\omega \geq 0$, we have $c_{i}(t+\omega)=c_{i}(t)$, $a_{i j}(t+\omega)=a_{i j}(t), b_{i j}(t+\omega)=b_{i j}(t), \alpha_{i j}(t+\omega)=\alpha_{i j}(t)$, $\beta_{i j}(t+\omega)=\beta_{i j}(t), T_{i j}(t+\omega)=T_{i j}(t), H_{i j}(t+\omega)=H_{i j}(t)$, $v_{i}(t+\omega)=v_{i}(t), I_{i}(t+\omega)=I_{i}(t)$, and $\tau_{i}(t+\omega)=\tau_{i}(t)$ for $t \geq t_{0}$. Thus, the sufficient conditions in Theorem 6 are satisfied.

Remark 8. If $I_{k}(\cdot)=0$, the model (1) is changed into

$$
\begin{aligned}
\frac{\partial u_{i}(t, x)}{\partial t}= & \sum_{l=1}^{m} \frac{\partial}{\partial x_{l}}\left(D_{i} \frac{\partial u_{i}(t, x)}{\partial x_{l}}\right) \\
& -c_{i}(t) u_{i}(t, x)+\sum_{j=1}^{n} a_{i j}(t) f_{j}\left(u_{j}(t, x)\right) \\
& +\sum_{j=1}^{n} b_{i j}(t) v_{j}(t)+J_{i}(t) \\
& +\bigwedge_{j=1}^{n} \alpha_{i j}(t) g_{j}\left(u_{j}\left(t-\tau_{j}(t), x\right)\right) \\
& +\bigvee_{j=1}^{n} \beta_{i j}(t) g_{j}\left(u_{j}\left(t-\tau_{j}(t), x\right)\right)
\end{aligned}
$$




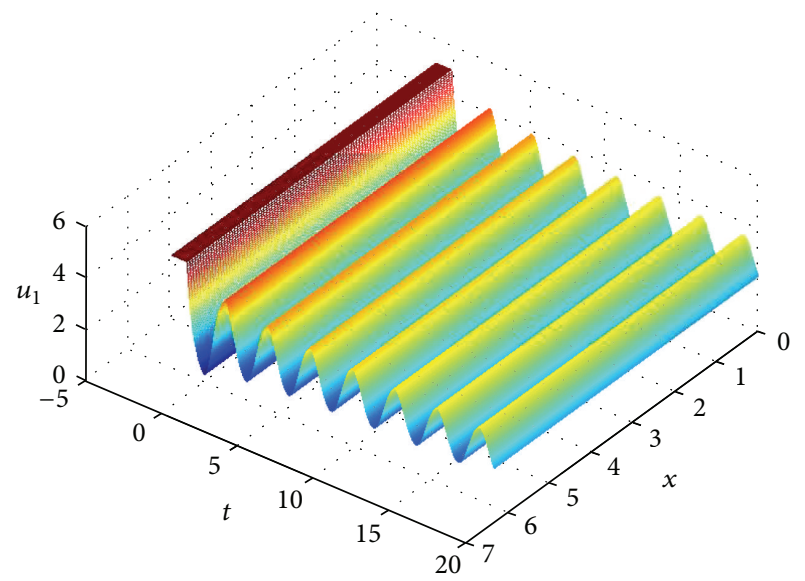

FigURE 1: State response $u 1(t, x)$ of model (44) without impulsive effects.

$$
\begin{gathered}
+\bigwedge_{j=1}^{n} T_{i j}(t) v_{j}(t)+\bigvee_{j=1}^{n} H_{i j}(t) v_{j}(t), \\
t \neq t_{k}, \quad x \in \Omega, \\
\frac{\partial u_{i}(t, x)}{\partial n}=0, \quad t \geq t_{0}, \quad x \in \partial \Omega, \\
u_{i}\left(t_{0}+s, x\right)=\psi_{i}(s, x), \quad-\tau_{j} \leq s \leq 0, x \in \Omega,
\end{gathered}
$$

which has been discussed in [22]. As Song and Wang have pointed out, the model (43) is more general than some wellstudied fuzzy neural networks. For example, when $c_{i}(t)>$ $0, a_{i j}(t), b_{i j}(t), \alpha_{i j}(t), \beta_{i j}(t), T_{i j}(t), H_{i j}(t), v_{i}(t)$, and $I_{i}(t)$ are all constants, the model in (43) reduces the model which has been studied by Huang [19]. Moreover, if $D_{i}=0, \tau_{i}(t)=0$, $f_{i}(\theta)=g_{i}(\theta)=(1 / 2)(|\theta+1|-|\theta-1|),(i=1, \ldots, n)$, then model $(42)$ covers the model studied by Yang et al. $[4,5]$ as a special case. If $D_{i}=0$ and $\tau_{j}(t)$ is assumed to be differentiable for $i, j=1,2, \ldots, n$, then model (43) can be specialized to the model investigated in Liu and Tang [12] and Yuan et al. [13]. Obviously, our results are less conservative than that of the above-mentioned literature, because they do not consider impulsive effects.

\section{Numerical Examples}

Example 9. Consider a two-neuron FIRDDCNN model:

$$
\begin{aligned}
\frac{\partial u_{i}(t, x)}{\partial t}= & \sum_{l=1}^{m} \frac{\partial}{\partial x_{l}}\left(D_{i} \frac{\partial u_{i}(t, x)}{\partial x_{l}}\right) \\
& -c_{i}(t) u_{i}(t, x)
\end{aligned}
$$

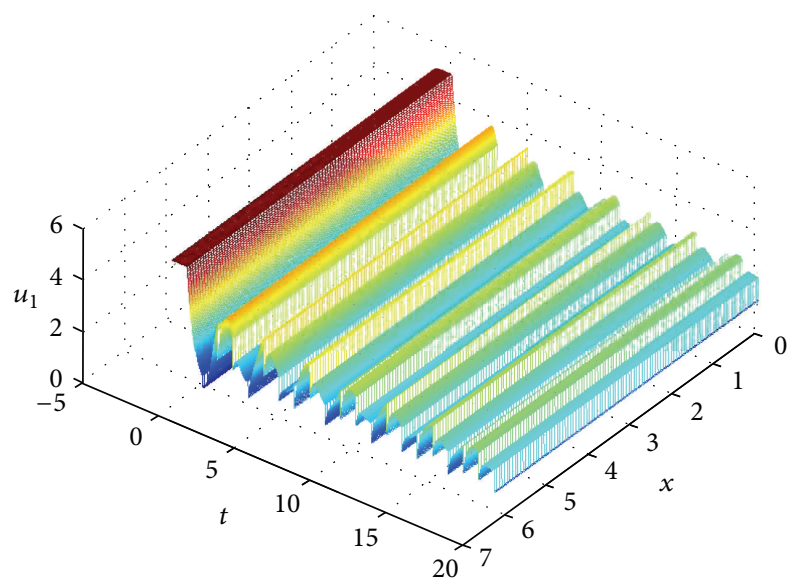

FIGURE 2: State response $u 1(t, x)$ of model (44) with impulsive effects.

$$
\begin{aligned}
& +\sum_{j=1}^{2} a_{i j}(t) f_{j}\left(u_{j}(t, x)\right) \\
& +\sum_{j=1}^{2} b_{i j}(t) v_{j}(t)+J_{i}(t) \\
& +\bigwedge_{j=1}^{2} \alpha_{i j}(t) g_{j}\left(u_{j}\left(t-\tau_{j}(t), x\right)\right) \\
& +\bigvee_{j=1}^{2} \beta_{i j}(t) g_{j}\left(u_{j}\left(t-\tau_{j}(t), x\right)\right) \\
& +\bigwedge_{j=1}^{2} T_{i j}(t) v_{j}(t)+\bigvee_{j=1}^{2} H_{i j}(t) v_{j}(t), \\
& t \neq t_{k}, x \in \Omega, \\
& u_{i}\left(t_{k}^{+}, x\right)=\left(1-\gamma_{i k}\right) u_{i}\left(t_{k}^{-}, x\right) \text {, } \\
& t=t_{k}, k \in \mathbb{Z}_{+}, x \in \Omega, \\
& \frac{\partial u_{i}(t, x)}{\partial n}=0, \quad t \geq t_{0}, x \in \partial \Omega, \\
& u_{i}\left(t_{0}+s, x\right)=\psi_{i}(s, x) \text {, } \\
& -\tau_{j} \leq s \leq 0, x \in \Omega,
\end{aligned}
$$

where $i=1$, 2. $c_{1}(t)=26, c_{2}(t)=20.8, a_{11}(t)=-1-\cos (t)$, $a_{12}(t)=1+\cos (t), a_{21}(t)=1+\sin (t), a_{22}(t)=-1-\sin (t)$, $D_{1}=8, D_{2}=4, \partial u_{i}(t, x) / \partial n=0\left(t \geq t_{0}, x=0,2 \pi\right), \gamma_{1 k}=0.4$, $\gamma_{2 k}=0.2, \psi_{1}(\cdot)=\psi_{1}(\cdot)=5, b_{11}(t)=b_{21}(t)=\cos (t), b_{12}(t)=$ $b_{22}(t)=-\cos (t), J_{1}(t)=J_{2}(t)=1, H_{11}(t)=H_{21}(t)=\sin (t)$, $H_{12}(t)=H_{22}(t)=-1+\sin (t), T_{11}(t)=T_{21}(t)=-\sin (t)$, $T_{12}(t)=T_{22}(t)=2+\sin (t), \tau_{1}(t)=\tau_{2}(t)=1, f_{j}\left(u_{j}\right)=$ $u_{j}(t, x)(j=1,2), g_{j}\left(u_{j}(t-1, x)\right)=u_{j}(t-1, x) e^{-u_{j}(t-1, x)}(j=$ $1,2), \alpha_{11}(t)=-12.8, \alpha_{21}(t)=\alpha_{12}(t)=-1+\cos (t), \alpha_{22}(t)=$ $-10, \beta_{11}(t)=12.8, \beta_{12}(t)=-1+\sin (t)=\beta_{21}(t), \beta_{22}(t)=$ 


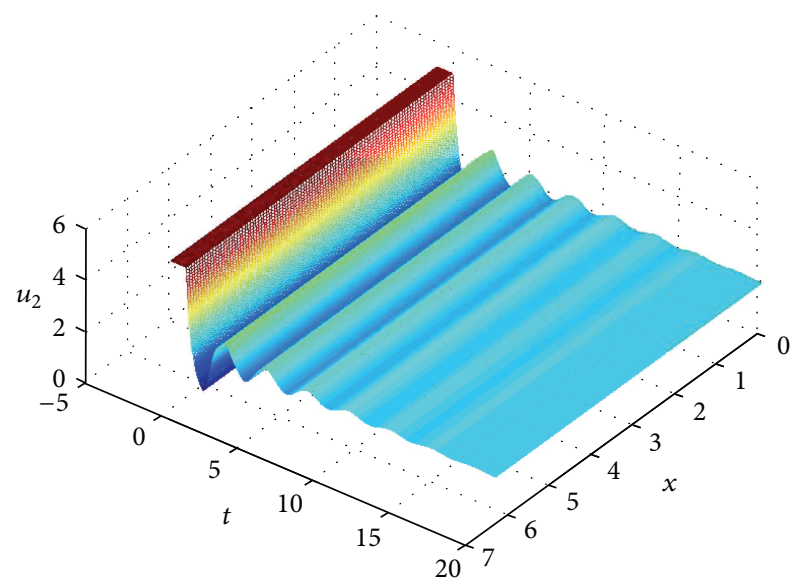

FIGURE 3: State response $u 2(t, x)$ of model (44) without impulsive effects.

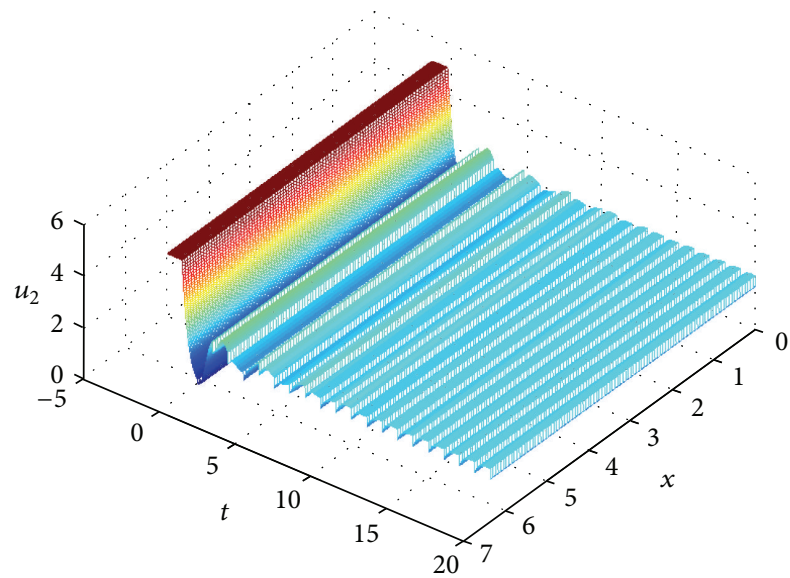

Figure 4: State response $u 2(t, x)$ of model (44) with impulsive effects.

$10, v_{j}(t)=\sin (t)$. We assume that there exists $q=6$ such that $t_{k}+2 \pi=t_{k+q}$. Obviously, $f_{1}, f_{2}, g_{1}$, and $g_{2}$ satisfy the assumption (H1) with $F_{1}=F_{2}=G_{1}=G_{2}=1$ and (H2) and (H3) are satisfied with a common positive period $2 \pi$

$$
\underline{C}-(\bar{A}+\bar{\alpha}+\bar{\beta}) F=\left[\begin{array}{ll}
\frac{2}{5} & 0 \\
0 & \frac{4}{5}
\end{array}\right]
$$

is a nonsingular $M$-matrix. The conditions of Theorem 6 are satisfied, hence there exists exactly one $2 \omega$-periodic solution of the model and all other solutions of the model converge exponentially to it as $t \rightarrow+\infty$. Furthermore, the exponential converging index can be calculated as $\lambda=0.021$, because here $\eta_{k}=1$ and $\eta=0$. The simulation results are shown in Figures $1,2,3$, and 4 , respectively.

\section{Conclusions}

In this paper, periodicity and global exponential stability of a class of FIRDDCNN model with variable both coefficients and delays have been investigated. By using Halanay's delay differential inequality, $M$-matrix theory, and analytic methods, some new sufficient conditions have been established to guarantee the existence, uniqueness, and global exponential stability of the periodic solution. Moreover, the exponential convergence rate index can be estimated. An example and its simulation have been given to show the effectiveness of the obtained results. In particular, the differentiability of the time-varying delays has been removed. The dynamic behaviors of fuzzy neural networks with the property of exponential periodicity are of great importance in many areas such as learning systems.

\section{Acknowledgments}

The authors would like to thank the editors and the anonymous reviewers for their valuable comments and constructive suggestions. This research is supported by the Natural Science Foundation of Guangxi Autonomous Region (no. 2012GXNSFBA053003), the National Natural Science Foundations of China (60973048, 61272077, 60974025, 60673101, and 60939003), National 863 Plan Project (2008 AA04Z401, 2009 AA043404), the Natural Science Foundation of Shandong Province (no. Y2007G30), the Scientific and Technological Project of Shandong Province (no. 2007GG3WZ04016), the Science Foundation of Harbin Institute of Technology (Weihai) (HIT (WH) 200807), the Natural Scientific Research Innovation Foundation in Harbin Institute of Technology (HIT. NSRIF. 2001120), the China Postdoctoral Science Foundation (2010048 1000), and the Shandong Provincial Key Laboratory of Industrial Control Technique (Qingdao University).

\section{References}

[1] L. O. Chua and L. Yang, "Cellular neural networks: theory," IEEE Transactions on Circuits and Systems, vol. 35, no. 10, pp. 1257$1272,1988$.

[2] L. O. Chua and L. Yang, "Cellular neural networks: applications," IEEE Transactions on Circuits and Systems, vol. 35, no. 10, pp. 1273-1290, 1988.

[3] T. Roska and L. O. Chua, "Cellular neural networks with non-linear and delay-type template elements and non-uniform grids," International Journal of Circuit Theory and Applications, vol. 20, no. 5, pp. 469-481, 1992.

[4] T. Yang, L. B. Yang, C. W. Wu, and L. O. Chua, "Fuzzy cellular neural networks: theory," in Proceedings of the 4th IEEE International Workshop on Cellular Neural Networks, and Their Applications (CNNA '96), pp. 181-186, June 1996.

[5] T. Yang and L.-B. Yang, "The global stability of fuzzy cellular neural network," IEEE Transactions on Circuits and Systems I, vol. 43, no. 10, pp. 880-883, 1996.

[6] T. Yang, L. B. Yang, C. W. Wu, and L. O. Chua, "Fuzzy cellular neural networks: applications," in Proceedings of the 4th IEEE International Workshop on Cellular Neural Networks, and Their Applications (CNNA '96), pp. 225-230, June 1996.

[7] S. T. Wang, K. F. L. Chung, and F. Duan, "Applying the improved fuzzy cellular neural network IFCNN to white blood cell detection," Neurocomputing, vol. 70, no. 7-9, pp. 1348-1359, 2007. 
[8] T. G. Barbounis and J. B. Theocharis, "A locally recurrent fuzzy neural network with application to the wind speed prediction using spatial correlation," Neurocomputing, vol. 70, no. 7-9, pp. 1525-1542, 2007.

[9] H. Zhao, "Global exponential stability and periodicity of cellular neural networks with variable delays," Physics Letters A, vol. 336, no. 4-5, pp. 331-341, 2005.

[10] S. Townley, A. Ilchmann, M. G. Weiss et al., "Existence and learning of oscillations in recurrent neural networks," IEEE Transactions on Neural Networks, vol. 11, no. 1, pp. 205-214, 2000.

[11] Z. Huang and Y. Xia, "Exponential periodic attractor of impulsive BAM networks with finite distributed delays," Chaos, Solitons \& Fractals, vol. 39, no. 1, pp. 373-384, 2009.

[12] Y. Liu and W. Tang, "Exponential stability of fuzzy cellular neural networks with constant and time-varying delays," Physics Letters A, vol. 323, no. 3-4, pp. 224-233, 2004.

[13] K. Yuan, J. Cao, and J. Deng, "Exponential stability and periodic solutions of fuzzy cellular neural networks with time-varying delays," Neurocomputing, vol. 69, no. 13-15, pp. 1619-1627, 2006.

[14] T. Huang, "Exponential stability of fuzzy cellular neural networks with distributed delay," Physics Letters A, vol. 351, no. 1-2, pp. 48-52, 2006.

[15] G. Bao, S. Wen, and Z. Zeng, "Robust stability analysis of interval fuzzy Cohen-Grossberg neural networks with piecewise constant argument of generalized type," Neural Networks, vol. 33, pp. 32-41, 2012.

[16] H. Zhao, N. Ding, and L. Chen, "Almost sure exponential stability of stochastic fuzzy cellular neural networks with delays," Chaos, Solitons \& Fractals, vol. 40, no. 4, pp. 1653-1659, 2009.

[17] W. Ding, M. Han, and M. Li, "Exponential lag synchronization of delayed fuzzy cellular neural networks with impulses," Physics Letters A, vol. 373, no. 8-9, pp. 832-837, 2009.

[18] L. Chen and H. Zhao, "Stability analysis of stochastic fuzzy cellular neural networks with delays," Neurocomputing, vol. 72, no. 1-3, pp. 436-444, 2008.

[19] T. Huang, "Exponential stability of delayed fuzzy cellular neural networks with diffusion," Chaos, Solitons \& Fractals, vol. 31, no. 3, pp. 658-664, 2007.

[20] J. Wang and J. G. Lu, "Global exponential stability of fuzzy cellular neural networks with delays and reaction-diffusion terms," Chaos, Solitons \& Fractals, vol. 38, no. 3, pp. 878-885, 2008.

[21] Q. Song and Z. Wang, "Dynamical behaviors of fuzzy reactiondiffusion periodic cellular neural networks with variable coefficients and delays," Applied Mathematical Modelling, vol. 33, no. 9, pp. 3533-3545, 2009.

[22] C. Wang, Y. kao, and G. Yang, "Exponential stability of impulsive stochastic fuzzy reaction-diffusion Cohen-Grossberg neural networks with mixed delays," Neurocomputing, vol. 89, pp. 55-63, 2012.

[23] T. Serrano-Gotarredona and B. Linares-Barranco, "LogDomain Implementation of Complex Dynamics ReactionDiffusion Neural Networks," IEEE Transactions on Neural Networks, vol. 14, no. 5, pp. 1337-1355, 2003.

[24] J. Liang and J. Cao, "Global exponential stability of reactiondiffusion recurrent neural networks with time-varying delays," Physics Letters A, vol. 314, no. 5-6, pp. 434-442, 2003.

[25] L. Wang and D. Xu, "Asymptotic behavior of a class of reactiondiffusion equations with delays," Journal of Mathematical Analysis and Applications, vol. 281, no. 2, pp. 439-453, 2003.
[26] L. Wang and D. Xu, "Global exponential stability of Hopfield reaction-diffusion neural networks with time-varying delays," Science in China. Series F, vol. 46, no. 6, pp. 466-474, 2003.

[27] Z. Yang and D. Xu, "Global dynamics for non-autonomous reaction-diffusion neural networks with time-varying delays," Theoretical Computer Science, vol. 403, no. 1, pp. 3-10, 2008.

[28] Y. G. Kao, C. C. Gao, and D. K. Wang, "Global exponential stability of reaction-diffusion Hopfield neural networks with continuously distributed delays," Mathematica Applicata, vol. 21, no. 3, pp. 457-462, 2008 (Chinese).

[29] Y.-G. Kao, J.-F. Guo, C.-H. Wang, and X.-Q. Sun, "Delaydependent robust exponential stability of Markovian jumping reaction-diffusion Cohen-Grossberg neural networks with mixed delays," Journal of the Franklin Institute, vol. 349, no. 6, pp. 1972-1988, 2012.

[30] H. Zhao and Z. Mao, "Boundedness and stability of nonautonomous cellular neural networks with reaction-diffusion terms," Mathematics and Computers in Simulation, vol. 79, no. 5, pp. 1603-1617, 2009.

[31] H. Zhao and G. Wang, "Existence of periodic oscillatory solution of reaction-diffusion neural networks with delays," Physics Letters A, vol. 343, no. 5, pp. 372-383, 2005.

[32] Q. Gan, "Exponential synchronization of stochastic CohenGrossberg neural networks with mixed time-varying delays and reaction-diffusion via periodically intermittent control," Neural Networks, vol. 31, pp. 12-21, 2012.

[33] V. Lakshmikantham, D. D. Bainov, and P. S. Simeonov, Theory of Impulsive Differential Equations, vol. 6 of Series in Modern Applied Mathematics, World Scientific, Teaneck, NJ, USA, 1989.

[34] D. D. Bainov and P. S. Simeonov, Impulsive Differential Equations: Periodic Solutions and Applications, Longman, Harlow, UK, 1993.

[35] X. Song, X. Xin, and W. Huang, "Exponential stability of delayed and impulsive cellular neural networks with partially Lipschitz continuous activation functions," Neural Networks, vol. 29-30, pp. 80-90, 2012.

[36] Z. Yang and D. Xu, "Existence and exponential stability of periodic solution for impulsive delay differential equations and applications," Nonlinear Analysis: Theory, Methods \& Applications, vol. 64, no. 1, pp. 130-145, 2006.

[37] X. Yang, X. Liao, D. J. Evans, and Y. Tang, "Existence and stability of periodic solution in impulsive Hopfield neural networks with finite distributed delays," Physics Letters A, vol. 343, no. 1-3, pp. 108-116, 2005.

[38] Y. Li and L. Lu, "Global exponential stability and existence of periodic solution of Hopfield-type neural networks with impulses," Physics Letters A, vol. 333, no. 1-2, pp. 62-71, 2004.

[39] Y. Li, L. Zhu, and P. Liu, "Existence and stability of periodic solutions of delayed cellular neural networks," Nonlinear Analysis: Real World Applications, vol. 7, no. 2, pp. 225-234, 2006.

[40] Z. H. Guan and G. Chen, "On delayed impulsive Hopfield neural networks," Neural Networks, vol. 12, no. 2, pp. 273-280, 1999.

[41] Z. H. Guan, J. Lam, and G. Chen, "On impulsive autoassociative neural networks," Neural Networks, vol. 13, no. 1, pp. 63-69, 2000.

[42] W. Ding, "Synchronization of delayed fuzzy cellular neural networks with impulsive effects," Communications in Nonlinear Science and Numerical Simulation, vol. 14, no. 11, pp. 3945-3952, 2009. 


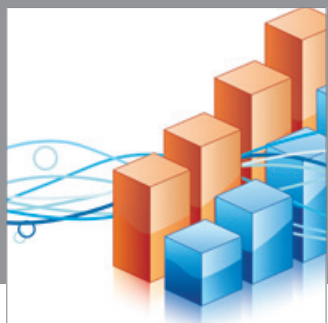

Advances in

Operations Research

mansans

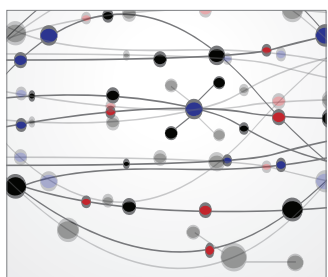

The Scientific World Journal
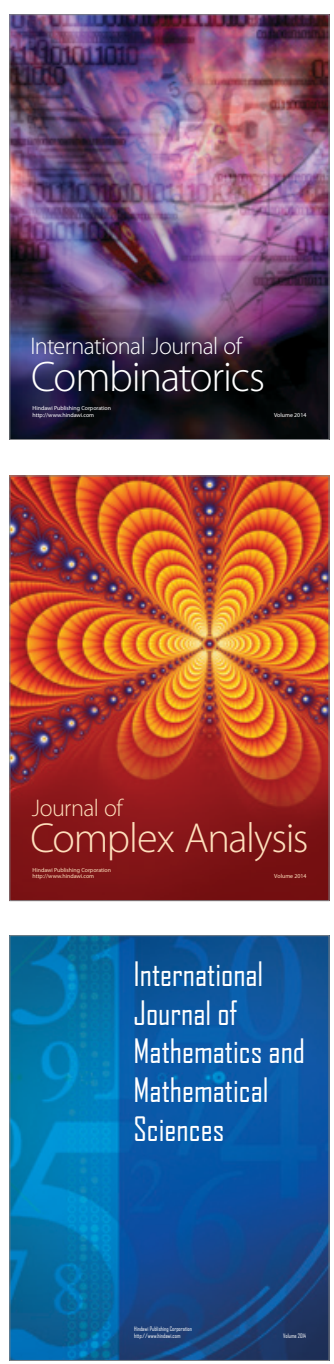
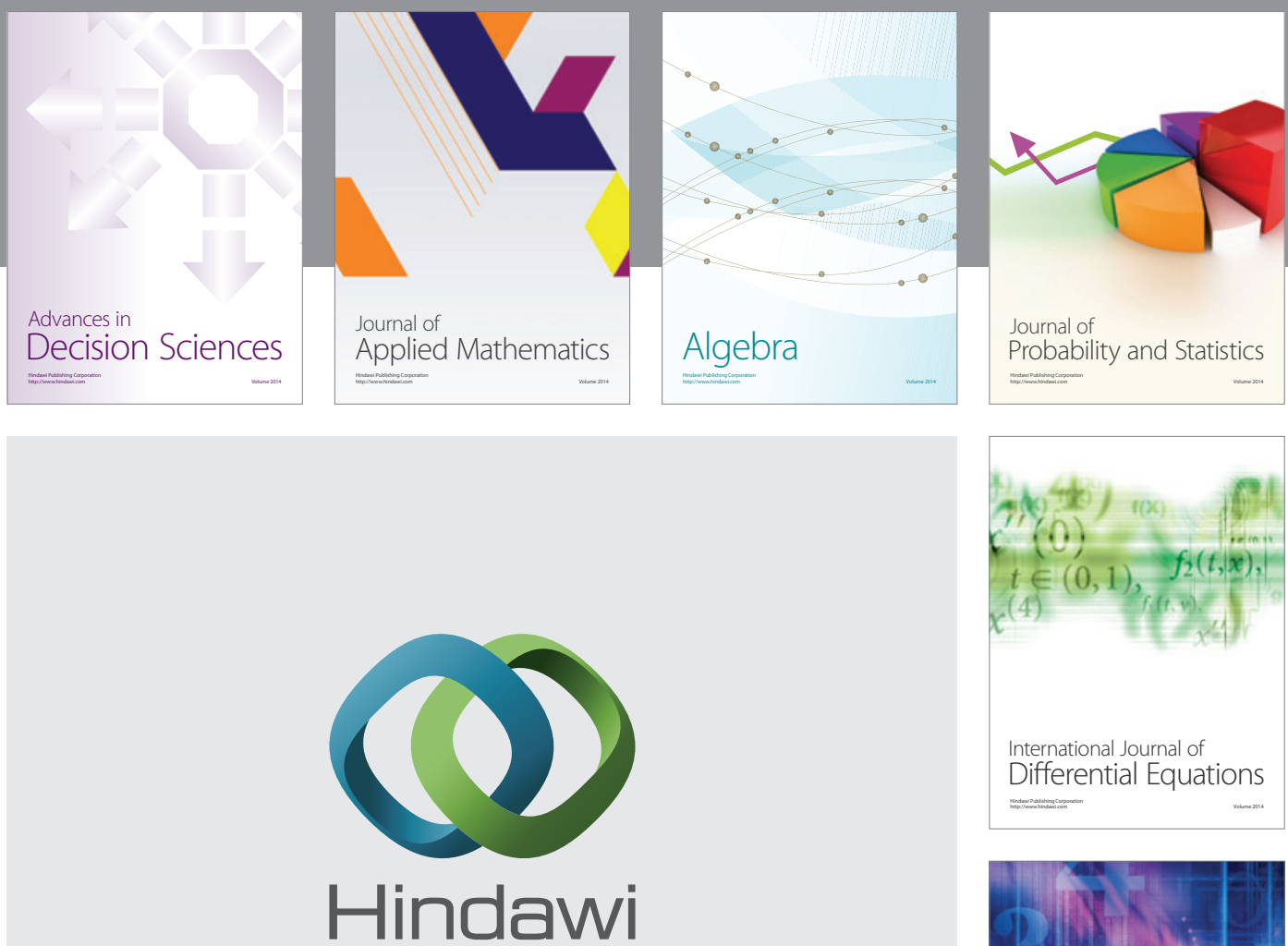

Submit your manuscripts at http://www.hindawi.com
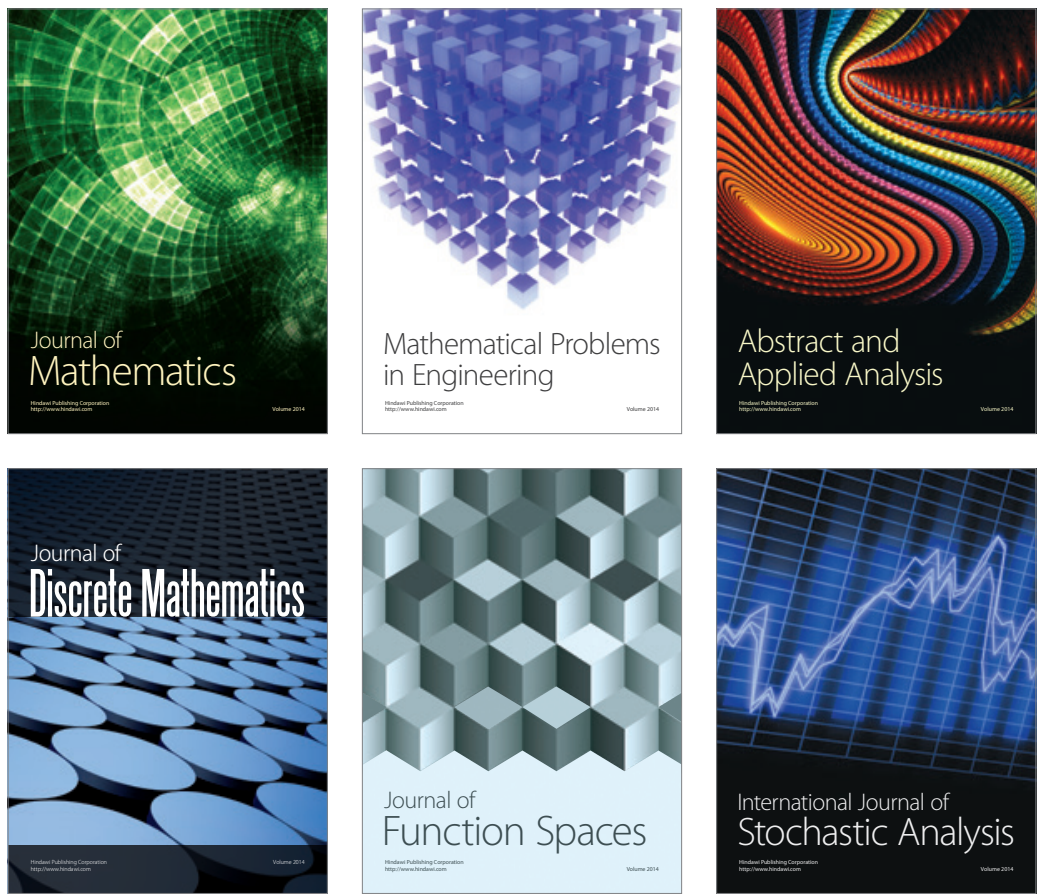

Journal of

Function Spaces

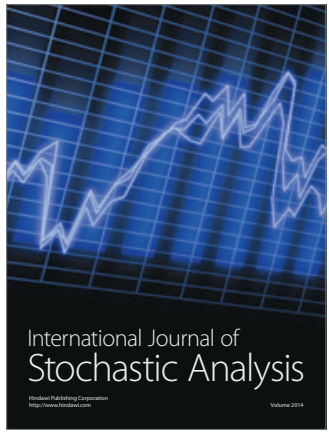

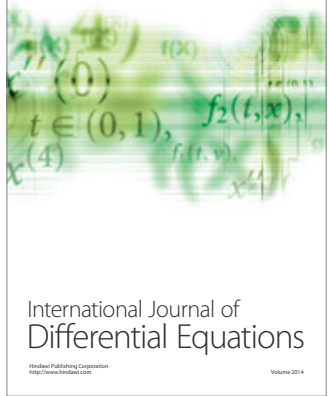
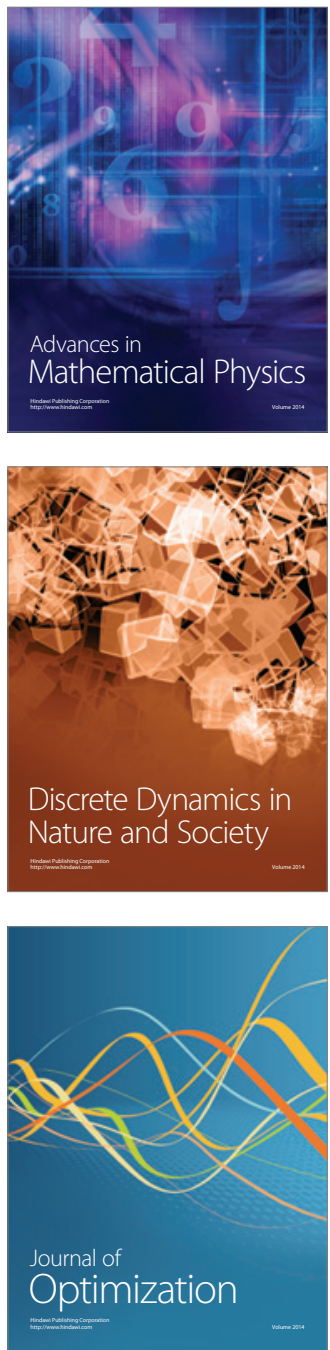